\title{
THE CONVEX FUNCTION DETERMINED BY A MULTIFUNCTION
}

\author{
M. CoOdey ANd S. Simons
}

\begin{abstract}
We shall show how each multifunction on a Banach space determines a convex function that gives a considerable amount of information about the structure of the multifunction. Using standard results on convex functions and a standard minimax theorem, we strengthen known results on the local boundedness of a monotone operator, and the convexity of the interior and closure of the domain of a maximal monotone operator. In addition, we prove that any point surrounded by (in a sense made precise) the convex hull of the domain of a maximal monotone operator is automatically in the interior of the domain, thus settling an open problem.
\end{abstract}

\section{INTRODUCTION}

We shall assume throughout this paper that $E$ is a nontrivial Banach space. We shall show how each multifunction $S: E \rightarrow 2^{E^{*}}$ with $D(S) \neq \emptyset$ determines a convex function $\chi_{S}: E \rightarrow \mathbb{R} \cup\{\infty\}$, and we shall also show that $\chi_{S}$ gives a considerable amount of information about the structure of $S$.

We define $\chi_{S}$ in Definition 2. Lemma 3 contains a technical result which will be useful later in the paper, and Lemma 4 is our main result about $\chi_{s}$. Our first application of Lemma 4 is in Theorem 6, in which we give a sufficient condition for $S$ to be locally bounded at a point of $\boldsymbol{E}$.

We next discuss the concept of an element $x$ of $E$ being "surrounded" by a subset $A$ of $E$. This concept is related to $x$ being an "absorbing point" of $A$, but differs in that we do not require that $x \in A$ (see [5, Definition 2.27(b), p.28]). Among other things, this difference will enable us to strengthen (in Theorem 12(b)) the result of Borwein and Fitzpatrick (see [1]) on the local boundedness of monotone operators.

Lemma 13(b) contains a result on the existence of elements of $E^{*}$, which we apply to maximal monotone operators in Theorem 14. Rockafellar proved in [7, Theorem 1, p.398] (see also [6, Theorem 1.9, p.6] that if $S$ is maximal monotone and int (co $D(S)) \neq$ $\emptyset$, then int $D(S)$ and $\overline{D(S)}$ are both convex. (As usual, "co" stands for "convex hull of".) In Theorem 14, we give more explicit results and prove that, in fact,

$$
\text { int } D(S)=\operatorname{int}\left(\operatorname{dom} \chi_{s}\right) \text { and } \overline{D(S)}=\overline{\operatorname{dom} \chi_{s}} \text {. }
$$

Received 21st September, 1995

Copyright Clearance Centre, Inc. Serial-fee code: 0004-9729/96 \$A2.00+0.00. 
The first of these results is true even if int $(\operatorname{co} D(S))=\emptyset$.

The equation "int $D(S)=\operatorname{sur}(\operatorname{co} D(S))$ " in Theorem 14 means the following: if $x \in E$ and,

$$
\text { for all } w \in E \backslash\{0\}, \text { there exists } \delta>0 \text { such that } x+\delta w \in \operatorname{co} D(S)
$$

then $x \in$ int $D(S)$. This result answers in the affirmative a question raised by Phelps (see $[5$, p.29] and $[6$, p.8]).

The analysis in this paper gives insight into the "relative difficulty" of the results on the convexity of int $D(S)$ and $\overline{\operatorname{dom} \chi s}$ on the one hand, and the results on local boundedness on the other. The former use Lemma 4 in full generality, while the latter use Lemma 4 only for $m=1$.

A word about tools. In Lemma 4 we use the standard result that a proper convex lower semicontinuous function on $E$ is continuous on the interior of its domain. In Lemma 13(b), we use a minimax theorem. In fact, we could have used the HahnBanach theorem or a sandwich theorem instead, but a minimax theorem gives the fastest proof. We use the following classical minimax theorem, which can be deduced from more general results of Fan (see [2]) or Sion (see [9]). Fan's proof used a separation theorem for sets in finite dimensional spaces, and Sion's proof used the KKM theorem, but Theorem 1 can easily be proved without any functional analysis or fixed-point related concepts. See, for instance, the proof of Sion's theorem given by Komiya in [4].

THEOREM 1. Let $X$ and $Y$ be nonempty compact convex subsets of topological vector spaces. Let $f: X \times Y \rightarrow \mathbb{R}$ be (separately) concave and upper semicontinuous on $X$ and convex and lower semicontinuous on $Y$. Then

$$
\max _{\boldsymbol{X}} \min _{\boldsymbol{Y}} f=\min _{\boldsymbol{Y}} \max _{\boldsymbol{X}} f
$$

THE CONVEX FUNCTION DETERMINED BY A MULTIFUNCTION

DEFINITION 2: If $m \geqslant 1$, let

$$
\sigma_{m}:=\left\{a=\left(a_{1}, \ldots, a_{m}\right): a_{1}, \ldots, a_{m} \geqslant 0, a_{1}+\cdots+a_{m}=1\right\} \subset \mathbb{R}^{m}
$$

If $S: E \rightarrow 2^{E^{*}}$ and $D(S) \neq \emptyset$, we define $\chi_{S}: E \rightarrow \mathbb{R} \cup\{\infty\}$ by

$$
\chi_{S}(w):=\sup _{m \geqslant 1,\left(y_{1}, y_{1}^{*}\right), \ldots,\left(y_{m}, y_{m}^{*}\right) \in G(S), a \in \sigma_{m}} \frac{\sum_{i} a_{i}\left\langle w-y_{i}, y_{i}^{*}\right\rangle}{1+\left\|\sum_{i} a_{i} y_{i}\right\|} .
$$

$\chi S$ is clearly convex and lower semicontinuous. (Here $G(S)$ stands for the graph of $S$.) 
In $[8]$, a function $\psi_{S}: E \rightarrow \mathbb{R} \cup\{\infty\}$ was defined by the formula

$$
\psi_{S}(w):=\sup _{\left(y, y^{*}\right) \in G(S)} \frac{\left\langle w-y, y^{*}\right\rangle}{1+\|y\|}
$$

which is the $m=1$ version of the formula used to define $\chi_{s}$. This was adequate for proving the convexity of int $D(S)$ and $\overline{D(S)}$ in the reflexive case, but it seems that the more complicated function $\chi_{S}$ is required in the general case.

The results on translation contained in Lemma 3 will enable us to simplify the computations in Theorems 6 and 14 considerably.

Lemma 3 . Let $S: E \rightarrow 2^{E^{*}}$ with $D(S) \neq \emptyset$, and $z \in E$. Define $T: E \rightarrow 2^{E^{*}}$ by

$$
T x:=S(x+z)
$$

Then:

(a) For all $w \in E$,

$$
\frac{\chi_{T}(w)}{1+\|z\|} \leqslant \chi_{S}(w+z) \leqslant(1+\|z\|) \chi_{T}(w)
$$

(b) $\operatorname{Dom} \chi_{T}=\operatorname{dom} \chi_{S}-z$.

(c) $D(T)=D(S)-z$.

(d) If $S$ is monotone or maximal monotone then so is $T$.

Proof: In (a), we shall prove the second inequality - the first inequality follows by replacing $z$ by $-z$ and interchanging the roles of $S$ and $T$.

Let $m \geqslant 1,\left(y_{1}, y_{1}^{*}\right), \cdots,\left(y_{m}, y_{m}^{*}\right) \in G(S)$ and $a \in \sigma_{m}$. Then

$$
\left(y_{1}-z, y_{1}^{*}\right), \cdots,\left(y_{m}-z, y_{m}^{*}\right) \in G(T)
$$

Thus, using the definition of $\chi_{T}(w)$,

$$
\begin{aligned}
\sum_{i} a_{i}\left\langle(w+z)-y_{i}, y_{i}^{*}\right\rangle & =\sum_{i} a_{i}\left\langle w-\left(y_{i}-z\right), y_{i}^{*}\right\rangle \\
& \leqslant\left(1+\left\|\sum_{i} a_{i}\left(y_{i}-z\right)\right\|\right) \chi_{T}(w) \\
& =\left(1+\left\|\sum_{i} a_{i} y_{i}-z\right\|\right) \chi_{T}(w) \\
& \leqslant\left(1+\left\|\sum_{i} a_{i} y_{i}\right\|+\|z\|\right) \chi_{T}(w) \\
& \leqslant\left(1+\left\|\sum_{i} a_{i} y_{i}\right\|\right)(1+\|z\|) \chi_{T}(w) .
\end{aligned}
$$


We obtain (a) by dividing by $\left(1+\left\|\sum_{i} a_{i} y_{i}\right\|\right)$, taking the supremum over $m,\left(y_{i}, y_{i}^{*}\right)$ and $a$, and using the definition of $\chi s(w+z)$.

(b) follows from (a), and (c) and (d) are immediate.

LEMma 4. Let $S: E \rightarrow 2^{E^{*}}$ with $D(S) \neq \emptyset$, and $0 \in \operatorname{int}\left(\operatorname{dom} \chi_{S}\right)$. Then there exist $\eta \in(0,1]$ and $P>0$ such that

$$
m \geqslant 1,\left(y_{1}, y_{1}^{*}\right), \cdots,\left(y_{m}, y_{m}^{*}\right) \in G(S) \text { and } a \in \sigma_{m}
$$

imply

$$
\sum_{i} a_{i}\left\langle y_{i}, y_{i}^{*}\right\rangle \geqslant \eta\left\|\sum_{i} a_{i} y_{i}^{*}\right\|-P\left(1+\left\|\sum_{i} a_{i} y_{i}\right\|\right)
$$

Proof: From [5, Proposition 3.3, p.39], there exist $\eta \in(0,1]$ and $P>0$ such that

$$
w \in E \text { and }\|w\| \leqslant \eta \quad \Longrightarrow \quad \chi_{S}(w) \leqslant P
$$

Thus,

$$
w \in E,\|w\| \leqslant \eta, m \geqslant 1,\left(y_{1}, y_{1}^{*}\right), \ldots,\left(y_{m}, y_{m}^{*}\right) \in G(S) \text { and } a \in \sigma_{m}
$$

imply that

$$
\sum_{i} a_{i}\left\langle w-y_{i}, y_{i}^{*}\right\rangle \leqslant P\left(1+\left\|\sum_{i} a_{i} y_{i}\right\|\right)
$$

that is to say,

$\sum_{i} a_{i}\left\langle y_{i}, y_{i}^{*}\right\rangle \geqslant \sum_{i} a_{i}\left\langle w, y_{i}^{*}\right\rangle-P\left(1+\left\|\sum_{i} a_{i} y_{i}\right\|\right)=\left\langle w, \sum_{i} a_{i} y_{i}^{*}\right\rangle-P\left(1+\left\|\sum_{i} a_{i} y_{i}\right\|\right)$

We complete the proof of Lemma 4 by taking the supremum of the right hand expression over all $w \in E$ such that $\|w\| \leqslant \eta$.

Definition 5: Let $S: E \rightarrow 2^{E^{*}}$ with $D(S) \neq \emptyset$, and $x \in E$. Following [6, Definition 1.8, p.5] we say that $S$ is locally bounded at $x$ if there exist $\delta, Q>0$ such that

$$
\left(y, y^{*}\right) \in G(S) \text { and }\|y-x\|<\delta \quad \Longrightarrow \quad\left\|y^{*}\right\| \leqslant Q .
$$

Note that this definition does not require that $x \in D(S)$.

Theorem 6. Let $S: E \rightarrow 2^{E^{*}}$ with $D(S) \neq \emptyset$. Then $S$ is locally bounded at each point of int (dom $\chi s)$.

ProOF: From the results on translation in Lemma 3, it suffices to prove that

$$
0 \in \operatorname{int}(\operatorname{dom} \chi s) \Longrightarrow S \text { is locally bounded at } 0
$$


So suppose that $0 \in \operatorname{int}(\operatorname{dom} \chi s)$. Let $\eta$ and $P$ be as in Lemma 4. From Lemma 4 with $m=1$,

$$
\left(y, y^{*}\right) \in G(S) \quad \Longrightarrow \quad \eta\left\|y^{*}\right\| \leqslant\left\langle y, y^{*}\right\rangle+P(1+\|y\|)
$$

So

$$
\begin{aligned}
\left(y, y^{*}\right) \in G(S) \text { and }\|y\| \leqslant \frac{\eta}{2} & \Longrightarrow \eta\left\|y^{*}\right\| \leqslant \frac{\eta}{2}\left\|y^{*}\right\|+P\left(1+\frac{\eta}{2}\right) \\
& \Longrightarrow\left\|y^{*}\right\| \leqslant \frac{3 P}{\eta} .
\end{aligned}
$$

Thus Definition 5 is satisfied with $\delta:=\eta / 2$ and $Q:=3 P / \eta$.

Since Theorem 6 only uses the $m=1$ version of Lemma 4 , it could in fact be strengthened to give the result that $S$ is locally bounded at each point of int (dom $\psi_{S}$ ) - see the comment following Definition 2.

\section{SURROUNDED POINTS AND SURROUNDING SETS}

Definition 7: Let $x \in E$ and $\boldsymbol{A} \subset \boldsymbol{E}$. We say that $\boldsymbol{A}$ surrounds $\boldsymbol{x}$ if, for each $w \in E \backslash\{0\}$, there exists $\delta>0$ such that $x+\delta w \in A$. Furthermore, we define

$$
\operatorname{sur} A:=\{x: x \in E, A \text { surrounds } x\} .
$$

We note that, in general, sur $A \not \subset A$. (Consider, for example, the case where $A$ is the circumference of a circle in the plane and $x$ is the centre of $A$.)

Lemma 8 provides some general culture concerning surrounding sets.

LEMma 8. Suppose that $C$ is a nonempty, convex subset of $E$. Then:

(a) $\operatorname{sur} C$ is convex.

(b) $\operatorname{sur} C \subset C$.

(c) $x \in \operatorname{sur} C$ if and only if, for each $w \in E$ there exists $\delta>0$ such that $x+[-\delta, \delta] w \subset C$, that is to say, $x$ is a radial point of $C$, (see $[3$, p.14]).

(d) If $\operatorname{sur} C \neq \emptyset$ then $\bar{C}=\overline{\operatorname{sur} C}$.

Proof: (a) Suppose that $x, y \in \operatorname{sur} C$ and $\theta \in[0,1]$. Let $w \in E \backslash\{0\}$, and pick $\delta_{1}, \delta_{2}>0$ such that $x+\delta_{1} w \in C$ and $y+\delta_{2} w \in C$. Define $\delta:=(1-\theta) \delta_{1}+\theta \delta_{2}$. Then, from the convexity of $C$,

$$
[(1-\theta) x+\theta y]+\delta w=(1-\theta)\left(x+\delta_{1} w\right)+\theta\left(y+\delta_{2} w\right) \in C .
$$

Since this holds for all $w \in E \backslash\{0\},(1-\theta) x+\theta y \in \operatorname{sur} C$, as required. 
(b) Suppose that $x \in \operatorname{sur} C$. Let $w \in E \backslash\{0\}$ and pick $\delta_{1}, \delta_{2}>0$ such that

$$
x+\delta_{1} w \in C \text { and } x-\delta_{2} w \in C
$$

Since $C$ is convex, $\left[x+\delta_{1} w, x-\delta_{2} w\right] \subset C$. In particular, $x \in C$.

(c) Suppose that $x \in \operatorname{sur} C$. Let $w \in E$. If $w=0$ then, by (b), $x+[-1,1] w=$ $\{x\} \subset C$. If $w \neq 0$, pick $\delta_{1}, \delta_{2}>0$ such that

$$
x+\delta_{1} w \in C \text { and } x-\delta_{2} w \in C
$$

Let $\delta=\min \left\{\delta_{1}, \delta_{2}\right\}$. Since $C$ is convex,

$$
x+[-\delta, \delta] w=[x-\delta w, x+\delta w] \subset\left[x+\delta_{1} w, x-\delta_{2} w\right] \subset C .
$$

The converse is immediate.

(d) Suppose that $x \in C$. Let $y \in \operatorname{sur} C$. We claim that

$$
\theta \in(0,1] \quad \Longrightarrow \quad(1-\theta) x+\theta y \in \operatorname{sur} C
$$

So let $\theta \in(0,1]$. Let $w \in E \backslash\{0\}$, and pick $\rho>0$ such that $y+\rho w \in C$. Define $\delta:=\rho \theta$. Then, from the convexity of $C$,

$$
[(1-\theta) x+\theta y]+\delta w=(1-\theta) x+\theta(y+\rho w) \in C
$$

Since this holds for all $w \in E \backslash\{0\},(1-\theta) x+\theta y \in \operatorname{sur} C$, as required. It now follows by letting $\theta \rightarrow 0+$ that $x \in \overline{\operatorname{sur} C}$. So we have proved that $C \subset \overline{\operatorname{sur} C}$, from which it follows immediately that $\bar{C} \subset \overline{\operatorname{sur} C}$. The reverse inclusion follows from (b), and this completes the proof of (d).

Let $E$ be infinite dimensional. Then there exists a discontinuous linear functional $L: E \rightarrow \mathbb{R}$. Let $C:=\{x \in E:|L x| \leqslant 1\}$. Then $C$ is convex and $0 \in \operatorname{sur} C$, but $0 \notin \operatorname{int} C$. The point of this simple example is to contrast the situation for general convex sets with that exhibited in Theorem 9.

THEOREM 9. Let $\emptyset \neq C \subset E$. Suppose that $\left\{F_{n}\right\}$ is an increasing sequence of closed convex sets such that $C=\bigcup_{n \geqslant 1} F_{n}$. Then $\operatorname{sur} C=\operatorname{int} C$.

PROOF: It suffices from a translation argument to show that

$$
0 \in \operatorname{sur} C \Longrightarrow 0 \in \operatorname{int} C
$$

Since $0 \in \operatorname{sur} C, E=\bigcup_{k \geqslant 1} k C$. So $E=\bigcup_{k, n \geqslant 1} k F_{n}$. By the Baire category theorem, there exist $n, k \geqslant 1$ such that int $k F_{n} \neq \emptyset$, from which int $F_{n} \neq \emptyset$. Choose $x \in \operatorname{int} F_{n}$. If 
$x=0$ then $0 \in \operatorname{int} F_{n} \subset \operatorname{int} C$. If $x \neq 0$ then, since $0 \in \operatorname{sur} C$, there exists $p>0$ such that $-x \in p C$, from which there exists $m \geqslant 1$ such that $-x \in p F_{m}$. Let $q=m \vee n$. Then

$$
x \in \operatorname{int} F_{q} \text { and } \frac{-x}{p} \in F_{q} .
$$

Using [3, 13.1(i), p.110], the convexity of $F_{q}$ implies $0 \in \operatorname{int} F_{q} \subset \operatorname{int} C$. This completes the proof of Theorem 9.

CoROLlary 10. Let $f: E \rightarrow \mathbb{R} \cup\{\infty\}$ be proper, convex and lower semicontinuous. Then $\operatorname{sur}(\operatorname{dom} f)=\operatorname{int}(\operatorname{dom} f)$.

Proof: This follows from Theorem 9 , with $F_{n}:=E\{f \leqslant n\}$.

\section{RESULTS FOR MONOTONE OPERATORS}

Lemma 11. Let $S: E \rightarrow 2^{E^{*}}$ be monotone, with $D(S) \neq \emptyset$. Then:

(a) $D(S) \subset \operatorname{co} D(S) \subset \operatorname{dom} \chi_{s}$.

(b) Let $m \geqslant 1,\left\{\left(y_{1}, y_{1}^{*}\right), \cdots,\left(y_{m}, y_{m}^{*}\right)\right\} \subset G(S)$ and $a \in \sigma_{m}$. Then

$$
\sum_{i} a_{i}\left\langle y_{i}, y_{i}^{*}\right\rangle \geqslant\left\langle\sum_{i} a_{i} y_{i}, \sum_{j} a_{j} y_{j}^{*}\right\rangle
$$

Proof: (a) Since dom $\chi_{S}$ is convex, it suffices to prove that

$$
D(S) \subset \operatorname{dom} \chi s
$$

To this end, let $w \in D(S)$. Pick $w^{*} \in S w$, and define $\beta:=\left\langle w, w^{*}\right\rangle \vee\left\|w^{*}\right\|$. Let $m \geqslant 1,\left(y_{1}, y_{1}^{*}\right), \cdots,\left(y_{m}, y_{m}^{*}\right) \in G(S)$, and $a \in \sigma_{m}$. Then, since $S$ is monotone,

$$
\begin{aligned}
\sum_{i} a_{i}\left\langle w-y_{i}, y_{i}^{*}\right\rangle & \leqslant \sum_{i} a_{i}\left\langle w-y_{i}, w^{*}\right\rangle \\
& =\left\langle w, w^{*}\right\rangle-\left\langle\sum_{i} a_{i} y_{i}, w^{*}\right\rangle \\
& \leqslant\left\langle w, w^{*}\right\rangle+\left\|\sum_{i} a_{i} y_{i}\right\|\left\|w^{*}\right\| \\
& \leqslant \beta\left(1+\left\|\sum_{i} a_{i} y_{i}\right\|\right) .
\end{aligned}
$$

Dividing by $1+\left\|\sum_{i} a_{i} y_{i}\right\|$, we obtain

$$
\frac{\sum_{i} a_{i}\left\langle\| w-y_{i}, y_{i}^{*}\right\rangle}{1+\left\|\sum_{i} a_{i} y_{i}\right\|} \leqslant \beta .
$$


Taking the supremum over $m \geqslant 1,\left(y_{1}, y_{1}^{*}\right), \cdots,\left(y_{m}, y_{m}^{*}\right) \in G(S)$ and $a \in \sigma_{m}$ we see that $\chi_{s}(w) \leqslant \beta$, which implies that $w \in \operatorname{dom} \chi_{s}$. This completes the proof of (11.1), and hence that of Lemma 11(a).

(b) follows from the following relations:

$$
\begin{aligned}
\sum_{i} a_{i}\left\langle y_{i}, y_{i}^{*}\right\rangle-\left\langle\sum_{i} a_{i} y_{i}, \sum_{j} a_{j} y_{j}^{*}\right\rangle & =\sum_{i, j} a_{i} a_{j}\left\langle y_{i}, y_{i}^{*}\right\rangle-\sum_{i, j} a_{i} a_{j}\left\langle y_{i}, y_{j}^{*}\right\rangle \\
& =\sum_{i, j} a_{i} a_{j}\left\langle y_{i}, y_{i}^{*}-y_{j}^{*}\right\rangle \\
& =\sum_{i<j} a_{i} a_{j}\left\langle y_{i}, y_{i}^{*}-y_{j}^{*}\right\rangle+\sum_{j<i} a_{i} a_{j}\left\langle y_{i}, y_{i}^{*}-y_{j}^{*}\right\rangle \\
& =\sum_{i<j} a_{i} a_{j}\left\langle y_{i}, y_{i}^{*}-y_{j}^{*}\right\rangle+\sum_{i<j} a_{i} a_{j}\left\langle y_{j}, y_{j}^{*}-y_{i}^{*}\right\rangle \\
& =\sum_{i<j} a_{i} a_{j}\left\langle y_{i}-y_{j}, y_{i}^{*}-y_{j}^{*}\right\rangle \geqslant 0 .
\end{aligned}
$$

ThEOREM 12. Let $S: E \rightarrow 2^{E^{*}}$ be monotone, with $D(S) \neq \emptyset$. Then:

(a) $\operatorname{sur} D(S) \subset \operatorname{sur}(\operatorname{co} D(S)) \subset \operatorname{sur}(\operatorname{dom} \chi s)$ $=\operatorname{int}(\operatorname{dom} \chi s) \supset \operatorname{int}(\operatorname{co} D(S)) \supset \operatorname{int} D(S)$.

(b) $S$ is locally bounded at each point of $\operatorname{sur}(\operatorname{co} D(S))$.

Proof: (a) It follows from Lemma 11(a) that $\operatorname{sur} D(S) \subset \operatorname{sur}(\operatorname{co} D(S)) \subset$ $\operatorname{sur}(\operatorname{dom} \chi s)$ and $\operatorname{int}\left(\operatorname{dom} \chi_{s}\right) \supset \operatorname{int}(\operatorname{co} D(S)) \supset \operatorname{int} D(S)$. Since $D(S) \neq \emptyset, \chi_{s}$ is proper so, from Corollary 10, $\operatorname{sur}(\operatorname{dom} \chi s)=\operatorname{int}(\operatorname{dom} \chi s)$.

(b) This is immediate from (a) and Theorem 6.

LemMA 13. Let $S: E \rightarrow 2^{E^{*}}$ be monotone with $D(S) \neq \emptyset, 0 \in \operatorname{int}\left(\operatorname{dom} \chi_{S}\right)$, and $\eta$ and $P$ be as in Lemma 4. Define $M:=P / \eta$. Now let $m \geqslant 1$ and $\left(y_{1}, y_{1}^{*}\right), \cdots,\left(y_{m}, y_{m}^{*}\right) \in G(S)$. Then:

(a) For all $a \in \sigma_{m}$,

$$
\sum_{i} a_{i}\left\langle y_{i}, y_{i}^{*}\right\rangle+M\left\|\sum_{i} a_{i} y_{i}\right\| \geqslant 0
$$

(b) There exists $z^{*} \in E^{*}$ such that

$$
\left\|z^{*}\right\| \leqslant M \text { and, for all } i=1, \ldots, m,\left\langle y_{i}, y_{i}^{*}-z^{*}\right\rangle \geqslant 0
$$


Proof: (a) Let $a \in \sigma_{m}$. If $\left\|\sum_{i} a_{i} y_{i}^{*}\right\|>M$ then, since $M=P / \eta \geqslant P$,

$$
\sum_{i} a_{i}\left\langle y_{i}, y_{i}^{*}\right\rangle+M\left\|\sum_{i} a_{i} y_{i}\right\| \geqslant \sum_{i} a_{i}\left\langle y_{i}, y_{i}^{*}\right\rangle+P\left\|\sum_{i} a_{i} y_{i}\right\|,
$$

from Lemma 4,

$$
\begin{aligned}
& \geqslant \eta\left\|\sum_{i} a_{i} y_{i}^{*}\right\|-P \\
& =\eta\left(\left\|\sum_{i} a_{i} y_{i}^{*}\right\|-M\right)>0,
\end{aligned}
$$

and (13.1) follows. If, on the other hand, $\left\|\sum_{i} a_{i} y_{i}^{*}\right\| \leqslant M$ then, from Lemma 11(b),

$$
\begin{aligned}
\sum_{i} a_{i}\left\langle y_{i}, y_{i}^{*}\right\rangle+M\left\|\sum_{i} a_{i} y_{i}\right\| & \geqslant\left\langle\sum_{i} a_{i} y_{i}, \sum_{i} a_{i} y_{i}^{*}\right\rangle+M\left\|\sum_{i} a_{i} y_{i}\right\| \\
& \geqslant M\left\|\sum_{i} a_{i} y_{i}\right\|-\left\|\sum_{i} a_{i} y_{i}\right\|\left\|\sum_{i} a_{i} y_{i}^{*}\right\| \\
& =\left(M-\left\|\sum_{i} a_{i} y_{i}^{*}\right\|\right)\left\|\sum_{i} a_{i} y_{i}\right\| \geqslant 0
\end{aligned}
$$

and (13.1) follows again. This completes the proof of Lemma 13(a).

(b) From Theorem 1,

$$
\begin{aligned}
\max _{\left\|z^{*}\right\| \leqslant M} \min _{a \in \sigma_{m}}\left[\sum_{i} a_{i}\left\langle y_{i}, y_{i}^{*}-z^{*}\right\rangle\right] & =\min _{a \in \sigma_{m}} \max _{\left\|z^{*}\right\| \in M}\left[\sum_{i} a_{i}\left\langle y_{i}, y_{i}^{*}-z^{*}\right\rangle\right] \\
& =\min _{a \in \sigma_{m}} \max _{\left\|z^{*}\right\| \leqslant M}\left[\sum_{i} a_{i}\left\langle y_{i}, y_{i}^{*}\right\rangle-\left\langle\sum_{i} a_{i} y_{i}, z^{*}\right\rangle\right] \\
& =\min _{a \in \sigma_{m}}\left[\sum_{i} a_{i}\left\langle y_{i}, y_{i}^{*}\right\rangle-\min _{\left\|z^{*}\right\| \leqslant M}\left\langle\sum_{i} a_{i} y_{i}, z^{*}\right\rangle\right] \\
& =\min _{a \in \sigma_{m}}\left[\sum_{i} a_{i}\left\langle y_{i}, y_{i}^{*}\right\rangle+M\left\|\sum_{i} a_{i} y_{i}\right\|\right] \geqslant 0,
\end{aligned}
$$

using (a). Thus there exists $z^{*} \in E^{*}$ such that $\left\|z^{*}\right\| \leqslant M$ and

$$
\text { for all } a \in \sigma_{m}, \sum_{i} a_{i}\left\langle y_{i}, y_{i}^{*}-z^{*}\right\rangle \geqslant 0 \text {. }
$$

We complete the proof of Lemma 13(b) by letting $a$ run through the vertices of $\sigma_{m}$.

Theorem 14. Let $S: E \rightarrow 2^{E^{*}}$ be maximal monotone. Then:

(a) $\operatorname{Sur} D(S)=\operatorname{sur}(\operatorname{co} D(S))=\operatorname{sur}(\operatorname{dom} \chi s)$

$$
=\operatorname{int}\left(\operatorname{dom} \chi_{S}\right)=\operatorname{int}(\operatorname{co} D(S))=\operatorname{int} D(S) \text {. }
$$


(b) If $\operatorname{sur}(\operatorname{co} D(S)) \neq \emptyset$ then

$$
\begin{aligned}
\overline{D(S)} & =\overline{\operatorname{co} D(S)}=\overline{\operatorname{dom} \chi s}=\overline{\operatorname{sur} D(S)}=\overline{\operatorname{sur}(\operatorname{co} D(S))} \\
& =\overline{\operatorname{sur}(\operatorname{dom} \chi s)}=\overline{\operatorname{int}\left(\operatorname{dom} \chi_{s}\right)}=\overline{\operatorname{int}(\operatorname{co} D(S))}=\overline{\operatorname{int} D(S)} .
\end{aligned}
$$

Proof: (a) We first prove that

$$
\operatorname{int}(\operatorname{dom} \chi s) \subset D(S)
$$

We can suppose that int $\left(\operatorname{dom} \chi_{s}\right) \neq \emptyset$, for otherwise there is nothing to prove. From the results on translation in Lemma 3 , it suffices to prove that

$$
0 \in \operatorname{int}\left(\operatorname{dom} \chi_{s}\right) \quad \Longrightarrow \quad 0 \in D(S)
$$

So suppose that $0 \in \operatorname{int}\left(\operatorname{dom} \chi_{s}\right)$. Let $M$ be as in Lemma 13. Then, for each finite subset $F$ of $G(S)$, the set

$$
\bigcap_{\left(y, y^{*}\right) \in F}\left\{z^{*}: z^{*} \in E^{*},\left\|z^{*}\right\| \leqslant M,\left\langle y, y^{*}-z^{*}\right\rangle \geqslant 0\right\}
$$

is nonempty. As $F$ runs, these sets are $w\left(E^{*}, E\right)$-compact and directed downwards, hence their intersection is nonempty. It follows that there exists $z^{*} \in E^{*}$ such that

$$
\text { for all }\left(y, y^{*}\right) \in G(S), \quad\left\langle y, y^{*}-z^{*}\right\rangle \geqslant 0
$$

Since $S$ is maximal monotone, this implies that $z^{*} \in S 0$, from which $0 \in D(S)$. This establishes (14.2), and hence (14.1). From (14.1), int $\left(\operatorname{dom} \chi_{S}\right) \subset \operatorname{int} D(S) \subset \operatorname{sur} D(S)$. The result follows from Theorem 12(a).

(b) From Lemma 11(a), $\overline{D(S)} \subset \overline{\operatorname{co} D(S)} \subset \overline{\operatorname{dom} \chi s}$. From (a), int (dom $\left.\chi_{s}\right) \neq \emptyset$. Thus, from $[3,13.1(\mathrm{i})]$ again, with $C:=\operatorname{dom} \chi_{s}$, and a second application of (a),

$$
\overline{\operatorname{dom} \chi s}=\overline{\operatorname{int}(\operatorname{dom} \chi s)}=\overline{\operatorname{int} D(S)} \subset \overline{D(S)}
$$

Thus we have proved that

$$
\overline{D(S)}=\overline{\operatorname{co} D(S)}=\overline{\operatorname{dom} \chi s}=\overline{\operatorname{int} D(S)} .
$$

The result now follows from a third application of (a). 


\section{REFERENCES}

[1] J. Borwein and S. Fitzpatrick, 'Local boundedness of monotone operators under minimal hypotheses', Bull. Austral. Math. Soc. 39 (1988), 439-441.

[2] K. Fan, 'Minimax theorems', Proc. Nat. Acad. Sci. U.S.A. 39 (1953), 42-47.

[3] J.L. Kelley, I. Namioka, and co-authors, Linear topological spaces (D. Van Nostrand Co., Inc., Princeton, Toronto, London, Melbourne, 1963).

[4] H. Komiya, 'Elementary proof for Sion's minimax theorem', Kodai Math. J. 11 (1988), 5-7.

[5] R.R. Phelps, Convex functions, monotone operators and differentiability, Lecture Notes in Mathematics 1364, (Second Edition) (Springer-Verlag, Berlin, Heidelberg, New York, 1993).

[6] R.R. Phelps, Lectures on maximal monotone operators, 2nd Summer School on Banach Spaces, Related Areas and Applications, Prague and Paseky, August 15-28, 1993 (Preprint, 30 pages.) TeX file: Banach space bulletin board archive: <ftp://ftp:@math.okstate.edu/pub/banach/phelpsmaxmonop.tex >. Posted November 1993.

[7] R.T. Rockafellar, 'Local boundedness of nonlinear, monotone operators', Michigan Math. J. 16 (1969), 397-407.

[8] S. Simons, 'The Range of a Monotone Operator', J. Math. Anal. Appl. 199 (1996), 176-201.

[9] M. Sion, 'On general minimax theorems', Pacific J. Math. 8 (1958), 171-176.

\footnotetext{
Department of Mathematics

University of California

Santa Barbara CA 93106-3080

United States of America
} 